Bull. Mater. Sci., Vol. 2, Number 3, August 1980, pp, 193-199, (C) Pridted in India,

\title{
Systematics in the electron transport and magnetic properties of $\mathrm{LnBO}_{3}$ perovskites*
}

\author{
P GANGULY and C N R RAO \\ Solid State and Structural Chemistry Unit, Indian Institute of Science, \\ Bangalore 560 012, India
}

MS received 9 April 1980

\begin{abstract}
Systematics in the electrical and magnetic properties of transition metal perovskites $\mathrm{LnBO}_{3}(\mathrm{Ln}=$ rare-earth ion, $\mathrm{B}=3 d$ transition metal) with the variation of $\mathrm{Ln}$ and $\mathrm{B}$ ions are reviewed. The electrical resistivity and activation energy of $\mathrm{LnBO}_{3}$ compounds increase with the decreasing size of the $\mathrm{Ln}^{3+}$ ion for a given $\mathrm{B}$ ion. The low-spin to high-spin transition temperature of $\mathrm{Co}^{3+}$ ion in $\mathrm{LnCoO}_{3}$ similarly increases with the decrease in size of $\mathrm{Ln}^{3+}$ while the magnetic ordering temperatures in $\mathrm{LnVO}_{3}, \mathrm{LnFeO}, \mathrm{LnCrO}_{3}$ and $\mathrm{LnSrCo}_{2} \mathrm{O}_{6}$ decreases with decreasing size of the rare-earth ion. These results may be understood in terms of the increasing acidity of the rare earth ion with decreasing size and the competition between the $\mathrm{Ln}^{3+}$ and the $\mathrm{B}^{3+}$ ions for covalency with the oxygen ions. The effect of this competition on the metal oxygen covalency and crystal field parameter is discussed in relation to the results obtained and Goodenough's phase diagram. The possibility of $d-f$ exchange interaction in $\mathrm{La}_{1-\mathrm{x}} \mathrm{Ln}_{\mathrm{x}} \mathrm{NiO}_{3}$ is also discussed in the light of ESR results.
\end{abstract}

Keywords. Perovskites; rare earth oxides; transition metal oxides; spin-state transitions; magnetic transitions.

\section{Introduction}

Oxide perovskites of the general formula $\mathrm{LnBO}_{3}(\mathrm{Ln}=$ rare-earth ion, and $\mathrm{B}=\mathrm{Ti}$, $\mathrm{V}, \mathrm{Cr}, \mathrm{Mn}, \mathrm{Fe}, \mathrm{Co}$, and $\mathrm{Ni}$ ) have been extensively studied by us over the past several years (see for example, Ganguly et al 1976). The $\mathrm{Ln}^{3+}$ ions occupy the body-centre position of the perovskite unit cell with the $\mathbf{B}$ ions occupying the corners. The volume of the unit cell decreases with the size of the rare-earth ion. This decrease is accompanied by deviations of the B-O-B angle from $180^{\circ}$ due to tilting of the octahedra. Other factors of importance in determining the properties of such perovskites are the acidity of the rare-earth ion and magnetic interactions between the rare-earth and transition metal ions.

From the one-electron energy diagram of Goodenough (1966)

$$
10 D q=\Delta_{\mathrm{x}}+\left(\lambda_{\sigma}^{2}-\lambda_{\pi}^{2}\right)\left(E_{\mathrm{m}}-E_{\mathrm{l}}\right)
$$

\footnotetext{
* Communication No, 66 from the Solid State and Structural Chemistry Unit.
} 
where $\triangle_{M}$ is the electrostatic contribution to the splitting of the $d$ orbitals of the $\mathrm{B}^{3+}$ ion, $\lambda_{\sigma}$ and $\lambda_{\pi}$ are the B-O covalent mixing parameters, and $\left(E_{\mathrm{M}}-E_{\mathrm{l}}\right)$ is the difference between the Madelung energy and the appropriate ionisation potential of the ion ; $\triangle_{\mathrm{M}}$ and $\left(E_{\mathrm{M}}-E_{\mathrm{l}}\right)$ may be assumed to be constant for a series of rareearth perovskites and hence $10 \mathrm{Dq}$ should depend on $\left(\lambda_{\sigma}^{2}-\lambda_{\pi}^{2}\right)$. The lanthanide ions compete with the $\mathrm{B}^{3+}$ ions for the covalency of the metal oxygen bond. The $\lambda_{\pi}$ covalent mixing parameter involving the $t_{2 \sigma}$ electron of the $\mathrm{B}$ ions and the $O_{2 p \pi}$ electrons are expected to change more drastically with change in the rare earth ion compared to $\lambda_{\sigma}$ as the $O_{2 p \pi}$ orbitals which are $\pi$ bonding with the metal ion are $\sigma$ bonding with the rare-earth ion. We should, therefore, expect that $\lambda_{\sigma}$ would be insensitive to the size or acidity of the rare-earth ion compared to $\lambda_{\pi}$ and the crystal field strength around the $B$ ion would increase as one goes down the rareearth series.

The most important effect of the rare-earth ion in the $\mathrm{LnBO}_{3}$ compounds is to affect the covalency of the B-O bond. Systematics in the electrical transport and magnetic properties of the $\mathrm{LnBO}_{3}$ compounds on reducing the size of the rareearth ion are expected from the variation in the covalency of the B-O bond. The

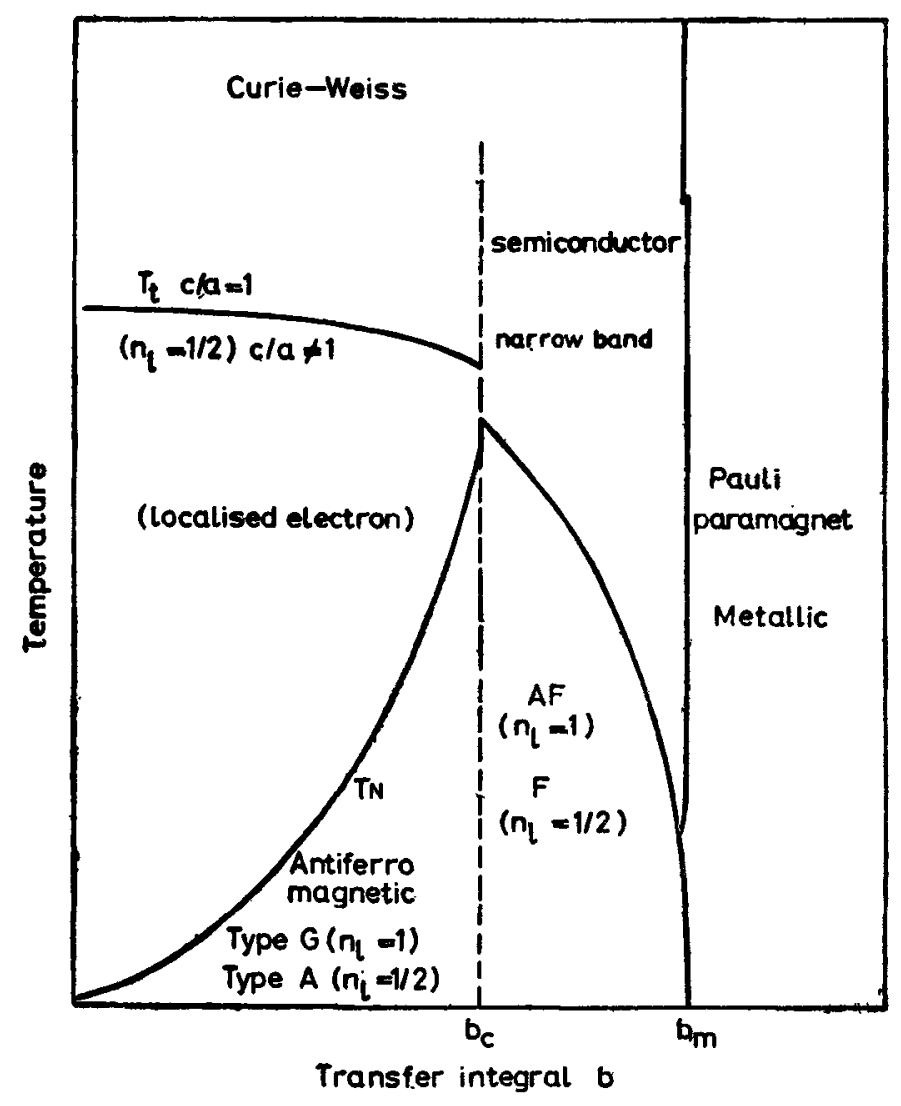

Figure 1. Goodenough's $b$ vs $T$ phase diagram. $b=$ transfer integra; $n_{l}=$ number of electrons per orbital per bond. 
phase diagram of Goodenough (figure 1) relating the magnetic ordering temperature as well as electrical transport properties to the cation-anion-cation exchange integral $b$ is appropriate for this purpose. As shown in figure 1 when $b<b_{a}$ the $d$ electrons (of the $B$ ions) are in the localised electron regime and super-exchange interactions are important. When $b_{\mathrm{c}}<b<b_{\mathrm{m}}$ spontaneous band magnetism due to electrons in strongly correlated bands appears while the region $b>b_{\mathrm{m}}$ corresponds to the itinerant electron regime. We present below some of our important results on the effect of the rare-earth ion on the electrical transport and magnetic properties of the $\mathrm{LnBO}_{3}$ perovskites in terms of the discussion presented above.

\section{Electrical transport properties}

Electrical resistivity data on the entire range of $\mathrm{LnBO}_{3}$ compounds studied in this laboratory are summarised in figure 2 . The resistivity generally increases with the decreasing size of the rare-earth ion (figure 3). The energy gap between the conduction band and the valence band therefore seems to be parallel to the changes in the crystal-field parameters.

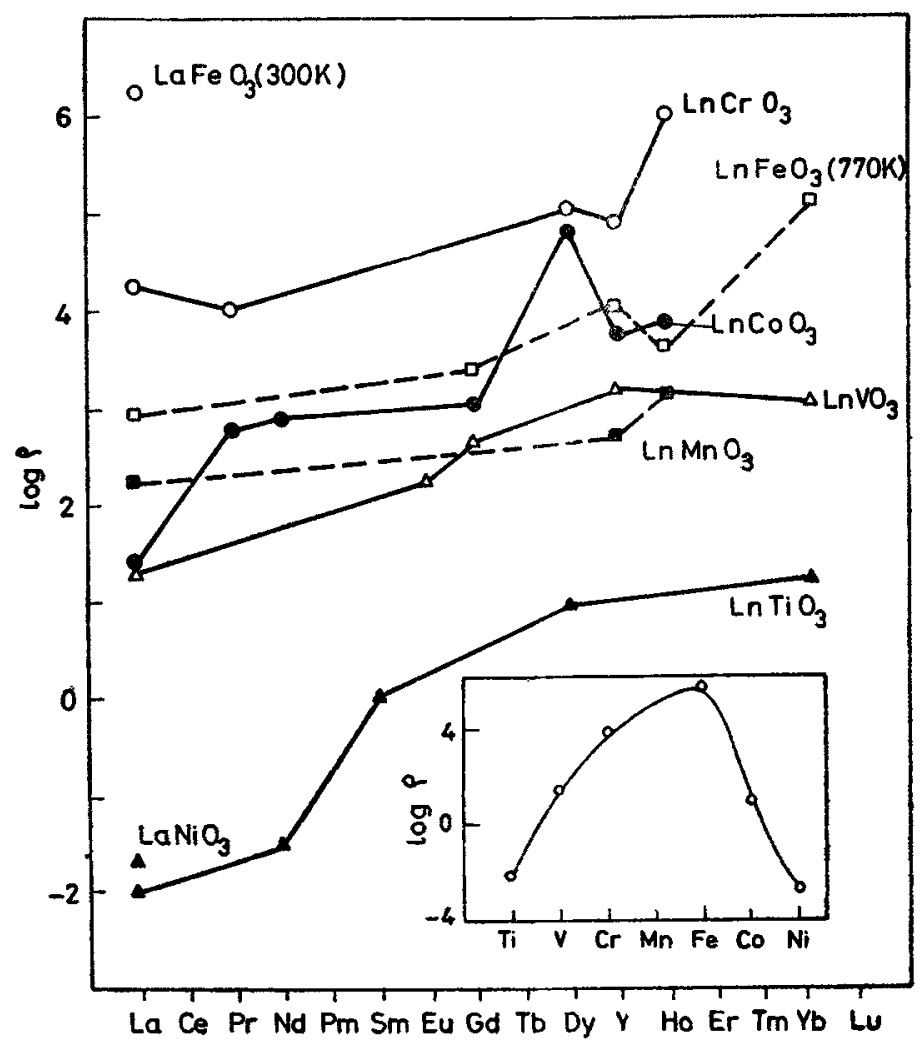

Figure 2. Changes in the room temperature electrical resistivity of the $\mathrm{LnBO}_{3}$ compounds with rare earth ion. Inset: room temperature electrical resistivity of $\mathrm{LaBO}_{3}$ compounds. 
It has been well documented that low-spin ions such as $\mathrm{Ti}^{3+}\left(t_{28}^{1}, e_{g}^{0}\right)$ and $\mathrm{Ni}^{3+}$ $\left(t^{6}{ }_{2 g}, e_{\mathrm{g}}^{1}\right)$ give rise to itinerant behaviour of the $d$ electrons while high-spin ions such as $\mathrm{Mn}^{3+}$ and $\mathrm{Fe}^{3+}$ give rise to localised $d$ electrons (Goodenough 1966). We indeed see that the room temperature resistivity of the $\mathrm{LaBO}_{3}$ compounds is a maximum when $\mathrm{B}=\mathrm{Fe}^{3+}$ (see inset of figure 2).

\section{Spin-state transitions}

The case of the cobaltites $\mathrm{LnCoO}_{3}$ is unique wherein both low-spin and high-spin states of $\mathrm{Co}^{3+}$ coexist giving rise to novel electrical and magnetic properties. There is a maximum in the $\chi^{-1}$ vs $T$ plot of $\mathrm{LaCoO}_{3}$ around $40 \mathrm{~K}$ followed by a minimum at $90 \mathrm{~K}$ and a plateau like region between 400 and $650 \mathrm{~K}$. These regions could be associated with spin-state transitions as shown in a theoretical model from this laboratory by Ramasesha et al (1979) in which a dynamical mixing of the highspin and low-spin state by an off-centre ion cage mode could give rise to a finite population of the high-spin ions at $0 \mathrm{~K}$. In the other $\mathrm{LnCoO}_{3}$ compounds where the $\mathrm{Ln}$ ion has unpaired $4 f$ electrons the contribution of the cobalt ions to the magnetic susceptibility $\left(\chi_{c o}\right)$ may be obtained by subtracting out the contribution

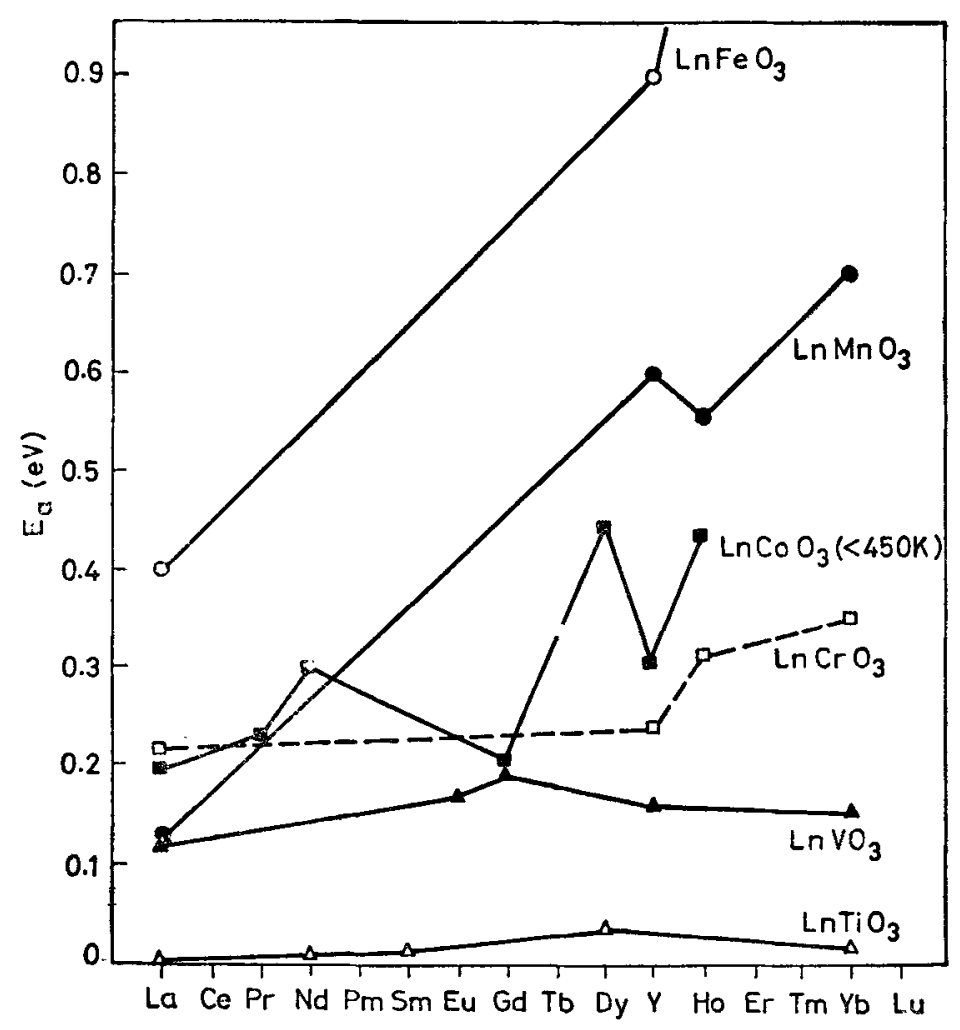

Figure 3. Variation in the activation energy $\left(E_{\mathrm{a}}\right)$ for electrical conduction of the $\mathrm{LnBO}_{3}$ compounds with rare-earth ion. 
to the susceptibility from the $\mathrm{Ln}^{3+}$ ions (as obtained from measurements on the isostructural $\mathrm{LnAlO}_{3}$ compounds). The $\chi_{\mathrm{co}}^{-1}$ vs $T$ plots of the various $\mathrm{LnCoO}_{3}$ compounds show maxima similar to that observed in $\mathrm{LaCoO}_{3}$ at $40 \mathrm{~K}$ and the shapes of these curves agree with the theoretical curves obtained by Ramasesha et al (1979). If we take the maximum in $\chi^{-1}$ to correspond to the spin state transition temperature $T_{\mathrm{s}}$ we see that $T_{\mathrm{s}}$ increases as we go down the rare-earth series as shown in figure 4. This trend reflects the increase in the crystal field around the $\mathrm{Co}^{3+}$ ion down the rare-earth series.

\section{Magnetic interactions}

We also show in figure 4 the antiferromagnetic ordering temperatures of the $\mathrm{LnBO}_{3}$ compounds $\left(\mathrm{B}=\mathrm{Fe}^{3+}, \mathrm{Cr}^{3+}, \mathrm{V}^{3+}\right)$. In all these cases the magnetic ordering tempe-

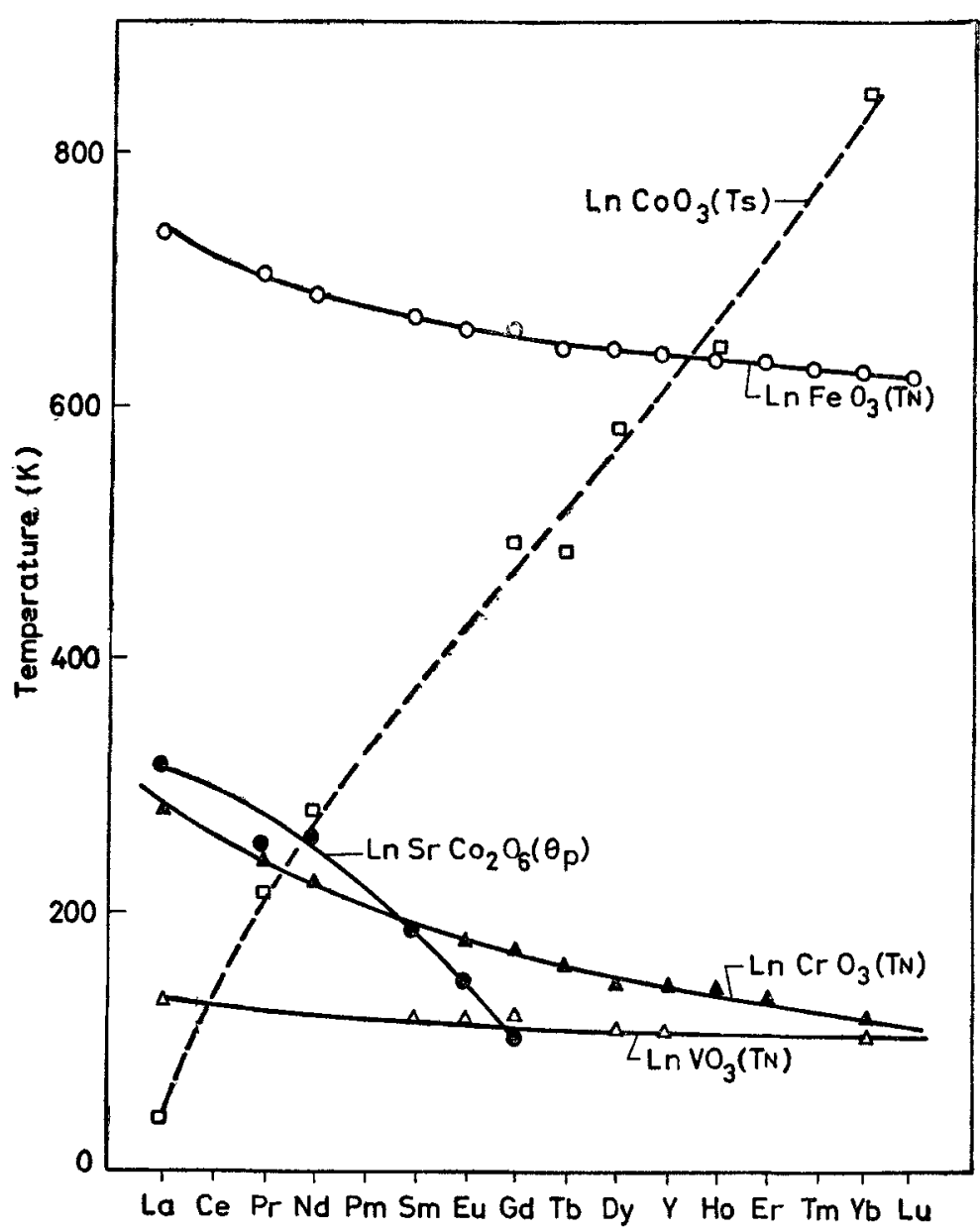

Figure 4. Variations in the spin-state transition temperature $T_{s}$ of $\mathrm{Co}^{3+}$ ions with rare-earth ion in $\mathrm{LnCoO}_{3}$ and in the magnetic ordering temperature $\left(T_{\mathrm{N}}\right)$ of $\mathrm{LnVO}_{3}$, $\mathrm{LnCrO}_{3}$ and $\mathrm{LnFeO}_{3}$ and paramagnetic Curie temperature $(\theta p)$ of $\mathrm{LnSrCo}_{2} \mathrm{O}_{6}$ with rare-earth ion. 
rature decreases monotonically with the size of the rare-earth ion. Since these solids behave as insulators the magnetic interaction is through the cation-anion-cation super-exchange mechanism. As seen from Goodenough's phase diagram (figure 1) the magnetic ordering temperature of the localised electron region is expected to decrease with $b$ and hence with the increasing acidity or the decreasing size of the rare earth ion.

We have examined the magnetic ordering temperature (Rao et al 1977) of the itenerant electron ferromagnets $\mathrm{LnSrCO}_{2} \mathrm{O}_{6}(\mathrm{Ln}=\mathrm{La}, \mathrm{Pr}, \mathrm{Nd}, \mathrm{Sn}, \mathrm{Eu}, \mathrm{Gd})$ where ferromagnetism arises from the $\mathrm{Co}^{3+}-\mathrm{O}-\mathrm{Co}^{4+}$ interaction. The paramagnetic Curie temperature $\theta p$ decreases as $\mathrm{Ln}$ is varied from $\mathrm{La}$ to $\mathrm{Gd}$ (figure 4). Hysteresis data show that the saturation magnetisation values at $77 \mathrm{~K}$ are almost independent of the rare-earth ion indicating that there is no contribution from the $4 f$ electrons to the magnetic moments in the ordered state. This is quite unlike the case of rare-earth ion garnets where the magnetic moments of the rare-earth ions are coupled to those of the iron ions. This seems to be a convincing demonstration that only the size of the rare-earth ion is important in determining the strength of the B-O-B interactions. That the acidity of the rare-earth ion is important is

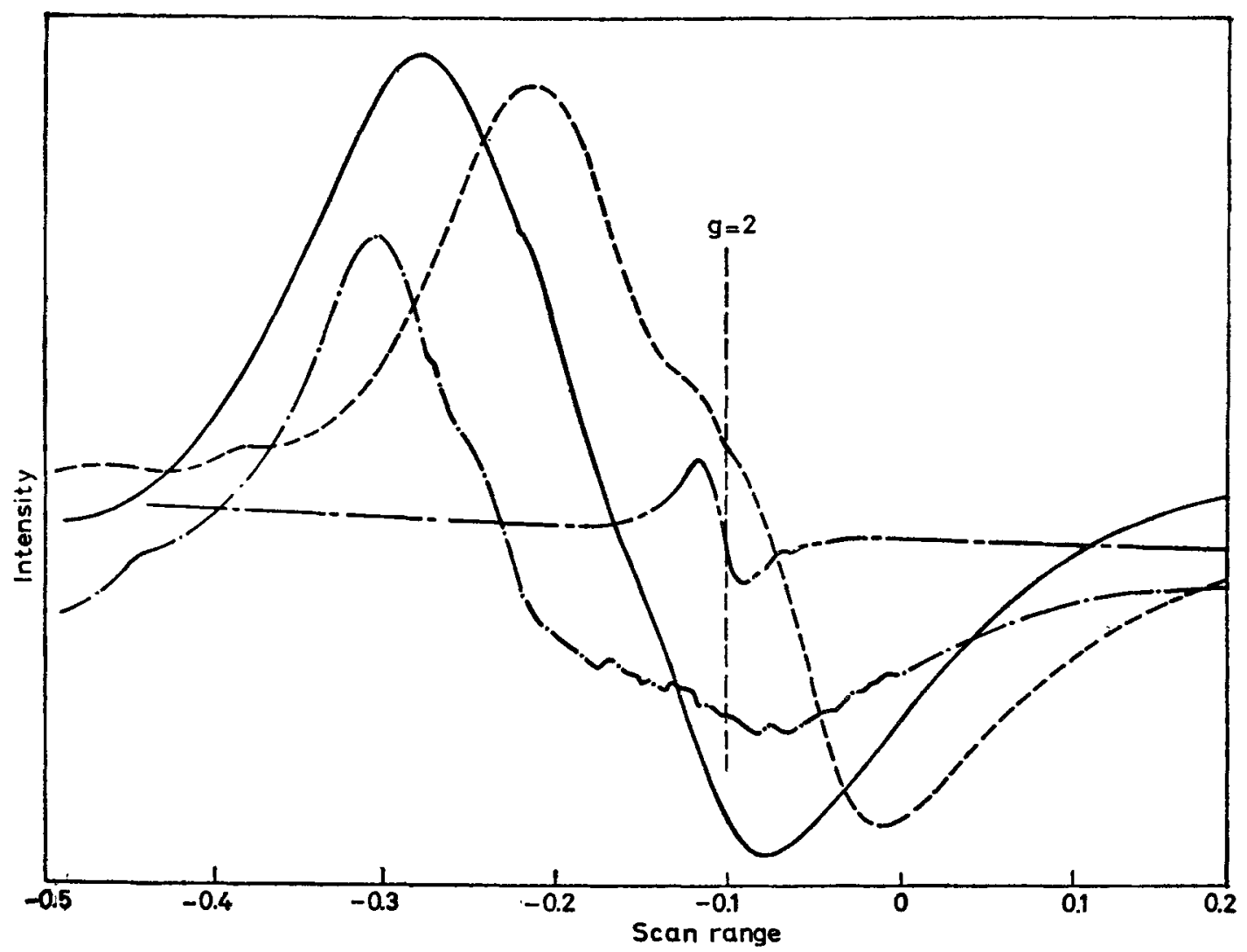

Figure 5. ESR spectra of some $\mathrm{La}_{0.98} \mathrm{Ln}_{0.02} \mathrm{NiO}_{3}$ compounds $(\mathrm{Ln}=\mathrm{Nd}, \mathrm{Gd}, \mathrm{Dy})$ at room temperature. Scan range $8 \times 1 \mathrm{kG}$, field set $4050 \mathrm{G}$, modulation amplitude $16 \mathrm{G}$, microwave frequency $9 \cdot 05 \mathrm{GHz}$. 
supported by the fact that the substitution of $\mathrm{Sr}$ in $\mathrm{LaSrCo}_{2} \mathrm{O}_{6}$ by a more acidic ion such as $\mathrm{Ca}^{2+}$ decreases $\theta p$ while a more basic ion such as $\mathrm{Ba}^{2+}$ increases $\theta p$.

We have recently carried out some preliminary investigations on the properties of $\mathrm{La}_{1-\mathrm{x}} \mathrm{Ln}_{\mathrm{x}} \mathrm{NiO}_{3}$ systems in order to examine $d-f$ exchange interaction. Since $\mathrm{LaNiO}_{3}$ has only itenerant $d$ electrons, localised $f$ electrons of $\mathrm{Ln}^{3+}$ ions could perturb the conduction $d$ electrons by a mechanism similar to the $s-d$ exchange interaction. Our preliminary results with $\mathrm{La}_{0 \cdot 98} \mathrm{Ln}_{0 \cdot 02} \mathrm{NiO}_{3}(\mathrm{Ln}=\mathrm{Nd}, \mathrm{Gd}, \mathrm{Dy}, \mathrm{Y})$ do not show any systematic changes in the magnetic susceptibility at room temperatures, although the $\mathrm{Nd}$ and $\mathrm{Dy}$ substituted compound show a field dependence of the magnetic susceptability at room temperatures which disappears at higher temperatures. ESR studies of powders of these compound show rather surprising results. Thus the ESR spectrum (figure 5) of $\mathrm{La}_{0 \cdot 98} \mathrm{Ln}_{0 \cdot 02} \mathrm{NiO}_{3}$ shows a single broad line (line width of nearly $2000 \mathrm{G}$ ) only when $\mathrm{Ln}^{3+}=\mathrm{Nd}^{3+}$, $\mathrm{Dy}^{3+}$ or $\mathrm{Gd}^{3+}$ but not with pure $\mathrm{LaNiO}_{3}$ or with the $\mathrm{Y}^{3+}$ substituted sample (although the size of the $\mathrm{Y}^{3+}$ ion is comparable to that of $\left.\mathrm{Dy}^{3+}\right)$. Such lines are not found in the corresponding $\mathrm{La}_{v \cdot 98} \mathrm{Ln}_{0 \cdot \cdot 2} \mathrm{AlO}_{3}$ samples. These results seem to support that the $\mathrm{Ni}^{3+}$ ions are localised around the rare earth ion and quite in contrast with our earlier discussion there seems to be some magnetic interactions between the $d$ electron of $\mathrm{Ni}^{3+}$ and the $f$ electrons of the $\mathrm{Ln}^{3+}$ ion. We have not yet ascertained whether this is peculiar to $\mathrm{Ni}^{3+}$ ions alone. (The $g$ values observed are different and quite close to those reported for the $\mathrm{Ln}^{3+}$ ions is other matters). The changes in the $g$ values and the changes in the shape clearly rule out the possibility of the signal being due to the presence of $\mathrm{Ni}^{2+}$ ions.

Another interesting experiment under way in this laboratory is to find the occurrence of Kondo anomaly or spin glass behaviour in $\mathrm{LaNiO}_{3}$ (and similar metallic hosts) doped with magnetic rare earth or transition metal ions. Such studies in oxide systems have not been reported hitherto.

\section{Acknowledgements}

The authors thank the Department of Science and Technology and the University Grants Commission, New Delhi, for financial support.

\section{References}

Ganguly P, Om Parkash and Rao C N R 1976 Phys. Status Solidi 36669 and references therein Goodenough J B 1966 J. Appl. Phys. 371415

Ramasesha S, Ramakrishnan T.V and Rao C N R 1979 J. Phys. C12 1307

Rao C N R, Om Parkash, Bahadur D, Ganguly P and Nagabushana S 1977 J. Solid State Chem. 22353 